\title{
A Heuristic Approach Based on Clarke-Wright Algorithm for Open Vehicle Routing Problem
}

\author{
Tantikorn Pichpibul and Ruengsak Kawtummachai \\ Faculty of Business Administration, Panyapiwat Institute of Management, 85/1 Moo 2, Chaengwattana Road, Bangtalad, \\ Pakkred, Nonthaburi 11120, Thailand \\ Correspondence should be addressed to Ruengsak Kawtummachai; ruengsak@yahoo.com
}

Received 15 July 2013; Accepted 8 October 2013

Academic Editors: A. Miné, D. Simson, and D. Talia

Copyright (C) 2013 T. Pichpibul and R. Kawtummachai. This is an open access article distributed under the Creative Commons Attribution License, which permits unrestricted use, distribution, and reproduction in any medium, provided the original work is properly cited.

\begin{abstract}
We propose a heuristic approach based on the Clarke-Wright algorithm $(\mathrm{CW})$ to solve the open version of the well-known capacitated vehicle routing problem in which vehicles are not required to return to the depot after completing service. The proposed CW has been presented in four procedures composed of Clarke-Wright formula modification, open-route construction, two-phase selection, and route postimprovement. Computational results show that the proposed CW is competitive and outperforms classical CW in all directions. Moreover, the best known solution is also obtained in $97 \%$ of tested instances (60 out of 62).
\end{abstract}

\section{Introduction}

The open vehicle routing problem (OVRP) was firstly solved by Sariklis and Powell [1] in their paper on distribution management problems. The characteristics of OVRP are similar to the capacitated vehicle routing problem (CVRP), which can be described as the problem of determining a set of vehicle routes to serve a set of customers with known geographical coordinates and known demands. A route represents a sequence of locations that a vehicle must visit. The distances between customer locations and between them and the depot are calculated or known in advance. For each route, the vehicle departs from the depot and returns to the depot after completing the service. The CVRP involves a single depot, a homogeneous fleet of vehicles, and a set of customers who require delivery of goods from the depot. The objective of the CVRP is to construct a feasible set of vehicle routes that minimizes the total traveling distance and/or the total number of vehicles used. Furthermore, the route must satisfy the constraints that each customer must be visited once, the demands of customers are totally satisfied, and the vehicle capacity is not exceeded for each route. In contrast, in the OVRP the companies either do not have their own vehicle available or the vehicles are inadequate to serve their customers. In this situation, the subcontracted vehicles will be hired from logistics outsourcing companies. Therefore, the transportation cost only depends on the traveling distance from depot to customers in which vehicles do not return to the depot and the maintenance cost does not occur. In another situation, the vehicles may return to the depot by following the same route in reverse order to collect items from the customers. The real-world case studies of OVRP are presented including a train plan model for British Rail freight services through the Channel Tunnel [2], the school bus routing problem in Hong Kong [3], the distribution of fresh meat in Greece [4], the distribution of a daily newspaper in the USA [5, 6], a lubricant distribution problem in Greece [7], and a mines material transport vehicle routing optimization in China [8].

The CVRP is one of the most important and widely studied problems in the area of combinatorial optimization. It comprises the traveling salesman problem (TSP) and the bin packing problem. The main distinction between OVRP and CVRP is that in CVRP each route is TSP which requires a Hamiltonian cycle [9], but in OVRP each route is a Hamiltonian path. Held and Karp [10] and Miller and Thatcher [11] have shown that the TSP is classified as NPhard (Non-deterministic Polynomial-time hard) problem. In addition, the Hamiltonian path has also been shown to be NP-hard [12]. Besides, the CVRP and OVRP are NP-hard 


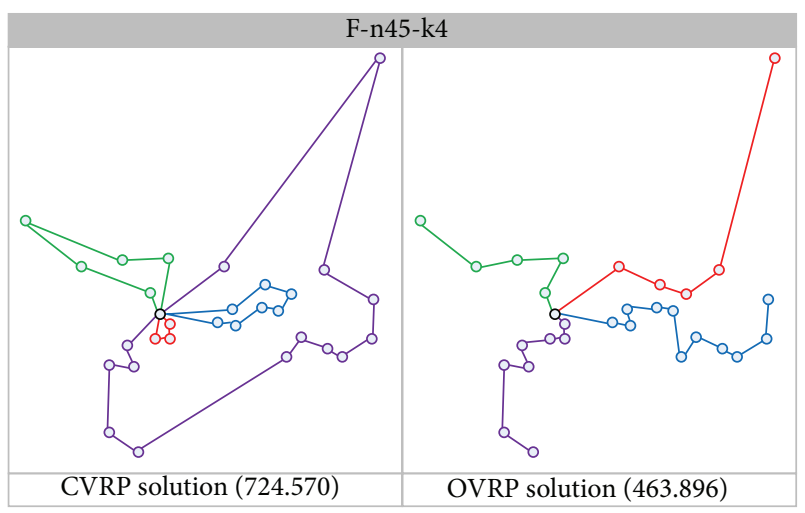

FIGURE 1: The difference of best known solutions between CVRP and OVRP.

[13-15]. In Figure 1, the best known solution for the OVRP is very different from the CVRP, and we also refer to Syslo et al. [12] that deleting the largest edge from a minimum Hamiltonian cycle does not necessarily yield the minimum Hamiltonian path in the network. The CVRP has attracted many researchers since Dantzig and Ramser [16] proposed the problem in 1959. Many efficient heuristics were presented to solve the CVRP. Conversely, from the early 1980s to the late 1990s, OVRP received very little attention in the operations research literature compared to CVRP. However, since 2000, several researchers have used various heuristics to solve OVRP, such as a tabu search $[14,17,18]$, an ant colony system [19], a variable neighborhood search [20], a particle swarm optimization [21], and a genetic algorithm [8].

The CW was proposed by Clarke and Wright [22] who introduced the savings concept which is based on the computation of savings for combining two customers into the same route. The CW is a widely known heuristic for solving the vehicle routing problem (VRP), and the applications of CW have continued since it was proposed in 1964. Improvements to the CW solution include proposed new parameters to the Clarke-Wright formulation composed of the nearest terminal $k$ for solving multidepot VRP [23], deleting $c_{j, 1}$ for solving OVRP [24], an estimate of the maximum savings value $s_{\max }$, and a penalty multiplier $\alpha$ for solving VRP with backhauls [25], route shape $\lambda$ for solving CVRP [26, 27], weight $\mu$ for asymmetric solving CVRP [28], the customer demand $v$ for solving CVRP [29], and the cosine value of polar coordinate angles of customers with the depot $\cos \theta_{i, j}$ for solving CVRP [30]. Second is improvements to the CW solution by proposed new probabilistic approaches to the $\mathrm{CW}$ procedure composed of the Monte Carlo simulation, cache, and splitting techniques for solving CVRP [31, 32], the two-phase selection and route postimprovement for solving CVRP $[33,34]$. Based on our review, there are very few works available in the literatures to modify the $\mathrm{CW}$ for solving OVRP (only Bodin et al. [24]). Therefore, this is our major contribution to improve the CW solution by using our simple, efficient, and competitive approach. In the proposed CW, we have modified the parallel version of CW to deal with OVRP and have combined this with a route postimprovement procedure which refers several neighborhood structures from the works of Subramanian et al. [35] and Groër et al. [36]. Moreover, the numerical experiment of $\mathrm{CW}$ for solving OVRP benchmark instances is also presented.

\section{The Proposed Clarke-Wright Algorithm}

Because CW is a heuristic algorithm, it cannot guarantee the best solution. Therefore, we introduce the modified version of the Clarke-Wright algorithm in which the parallel version of $\mathrm{CW}$ is implemented since it usually generates better results than the corresponding sequential version $[13,37]$. The flowchart of the proposed CW is given in Figure 2. First, the Euclidean distance matrix $\left(c_{i, j}\right)$ is calculated with the following equation:

$$
c_{i, j}=\sqrt{\left(x_{i}-x_{j}\right)^{2}+\left(y_{i}-y_{j}\right)^{2}},
$$

where $x_{i}, y_{i}$ and $x_{j}, y_{j}$ are the geographical locations of customer $i$ and $j$. Second, the savings value between customer $i$ and $j$ is calculated as

$$
s_{i, j}=c_{1, j}-c_{i, j}
$$

where $c_{1, j}$ is the traveling distance between depot and customer $j$ and $c_{i, j}$ is the traveling distance between customer $i$ and $j$. Equation (2) is modified by Bodin et al. [24] from the Clarke-Wright formulation which is shown in (3). After calculation, all savings values are collected in the savings list as follows:

$$
s_{i, j}=c_{1, i}+c_{j, 1}-c_{i, j} .
$$

Third, the values in the savings list are sorted in decreasing order. Finally, the route merging procedure starts from the top of the savings list (the largest $s_{i, j}$ ). Both customers $i$ and $j$ will be combined into the same route if the total demand does not exceed vehicle capacity and no route constraints exist. Each condition for route constraints is described by three cases of five customers as shown in Figure 3. In Figure 3(a), (1 nor 3 ) have already been assigned to a route (1-2-3). In Figure 3(b), exactly one of the two customers (2 or 4 ) has already been included in an existing route (1-2-3) and customer (2) is not interior to that route (a customer is interior to a route if it is not adjacent to the depot in the order of traversal of customers). In Figure 3(c), both customers ( 2 and 4$)$ have already been included in two different existing routes (1-2 and 3-4-5), and customer (4) is also interior to its route (3-4-5). The route merging procedure is repeated until no feasible merging in the savings list is possible. Furthermore, in case of nonrouted customers, each is assigned by a route that starts at the depot, visits the unassigned customer, and returns to the depot.

The proposed CW is an iterative improvement approach designed to find the global optimum solutions. It has been presented in four procedures consisting of Clarke-Wright formula modification, open-route construction, two-phase selection, and route postimprovement. The details of these procedures are shown below. 


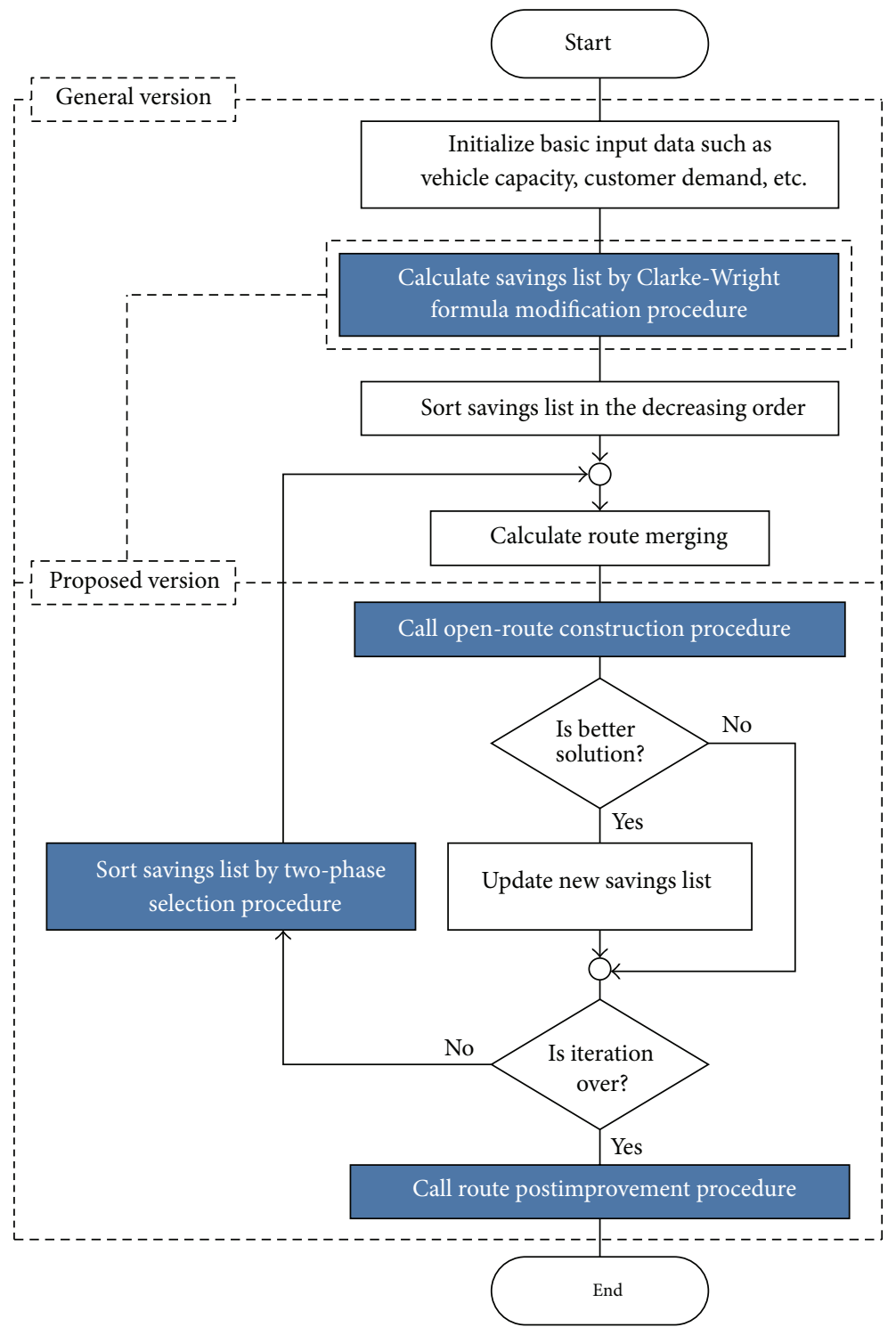

Figure 2: The flowchart of the proposed CW.

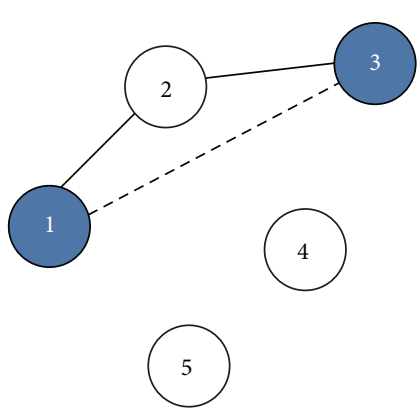

(a)

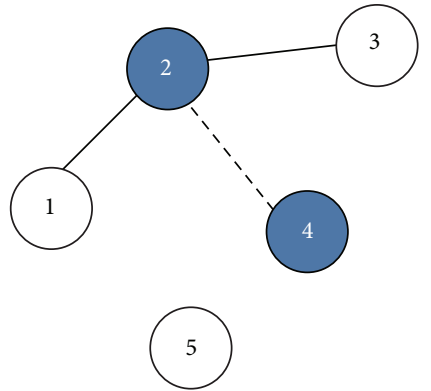

(b)

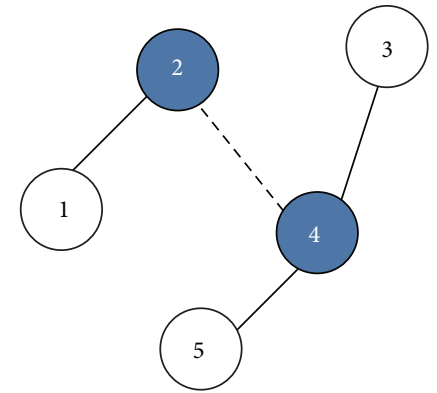

(c)

FIGURE 3: The route constraints of route merging procedure. 


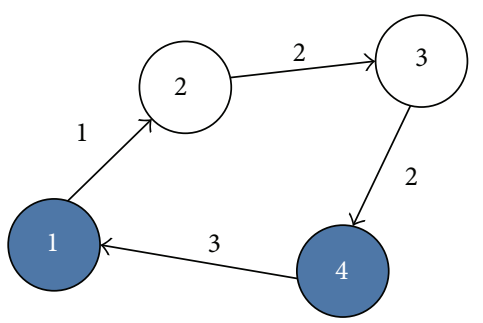

Solution $=1-2-3-4-1$

Value $=1+2+2+3=8$

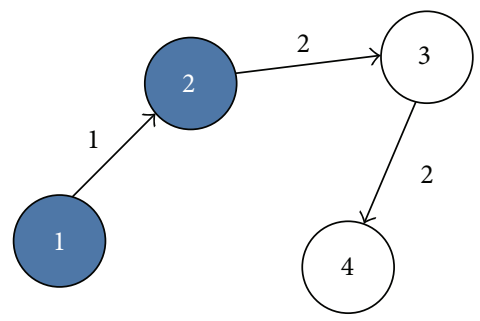

Solution $=1-2-3-4$

Value $=1+2+2=5$

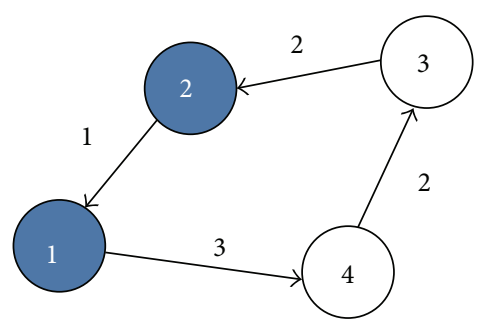

Solution $=1-4-3-2-1$

Value $=3+2+2+1=8$

(a)

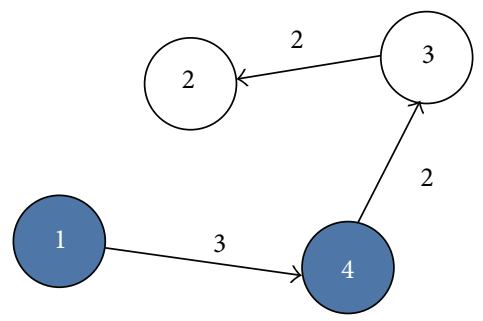

Solution $=1-4-3-2$

Value $=3+2+2=7$

(b)

FIgURE 4: The two possible CVRP and OVRP solutions.

2.1. The Clarke-Wright Formula Modification Procedure. Due to the latest improvement of CW for solving VRP that we mentioned in the literatures above, many authors proposed new parameters which we applied to this procedure. Gaskell [26] and Yellow [27] presented a route shape $(\lambda)$ parameter which controls the relative significance of direct arc between two customers. Their proposed savings formula is as follows:

$$
s_{i, j}=c_{1, i}+c_{j, 1}-\lambda c_{i, j}
$$

According to (2), we also modified (4) by deleting $c_{j, 1}$ for solving OVRP with the following equation:

$$
s_{i, j}=c_{1, j}-\lambda c_{i, j}
$$

The parameter could be varied as studied by Altinel and Öncan [29]. They used a simple enumerative approach to produce 8820 different solutions $(\lambda \in[0.1,2], \mu \in[0,2]$, $\nu \in[0,2])$. After that the best solution will be chosen. In order to avoid time-consuming iterative solutions, we have therefore applied only single parameter $(\lambda)$ to this procedure.

2.2. The Open-Route Construction Procedure. In order to solve CVRP by the CW after the route merging procedure, this procedure is needed to create the solution. Each route has to construct for the close route (Hamiltonian cycle) by assigning the first customer who starts at the depot, and the last customer who returns to the depot. As shown in Figure 4(a), the Euclidean distance represented in this paper is symmetric.
Therefore, the two possible CVRP solutions (1-2-3-4-1 and 1-4-3-2-1) are similar. In contrast, in OVRP each route has to construct for the open route (Hamiltonian path) by only assigning the first customer who starts at the depot. As shown in Figure 4(b), the two possible OVRP solutions (1-2-3-4 and 1-4-3-2) are very different. Consequently, in this procedure, the two possible OVRP solutions are constructed, and then the best solution will be selected as the OVRP solution.

2.3. The Two-Phase Selection Procedure. After the CW solution is produced from a standard savings list generated by sorting the savings values in the decreasing order, this savings list will be regenerated as a new one by sorting the savings values randomly with probability. Pichpibul and Kawtummachai [34] introduced the two-phase selection procedure for CVRP. In this paper, we adjust this procedure to deal with an OVRP by based on an operation of the genetic algorithm [38]. Figure 5(a) shows a genetic representation of chromosomes for ten savings values. The savings list is represented by one chromosome, and each gene represents the savings value between customers $i$ and $j$. In the first iteration, the chromosome is the savings list sorted by the decreasing order, but in next iteration the chromosome is the savings list derived from the best one. In Figure 5(b), we select one gene from the top four genes $(T=4)$ in the chromosome by fitness proportionate selection or roulette wheel selection. Here $T$ is tournament size which is a random number between three and six. In order to create a roulette wheel, the selection probability $\left(p_{n}\right)$ and cumulative probability $\left(q_{n}\right)$ 


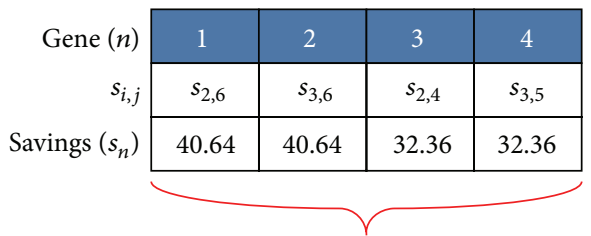

\begin{tabular}{|c|c|c|c|c|c|}
\hline 5 & 6 & 7 & 8 & 9 & 10 \\
\hline$s_{2,3}$ & $s_{4,6}$ & $s_{5,6}$ & $s_{2,5}$ & $s_{3,4}$ & $s_{4,5}$ \\
\hline 30.58 & 28.28 & 28.28 & 20.00 & 20.00 & 11.72 \\
\hline
\end{tabular}

(a)

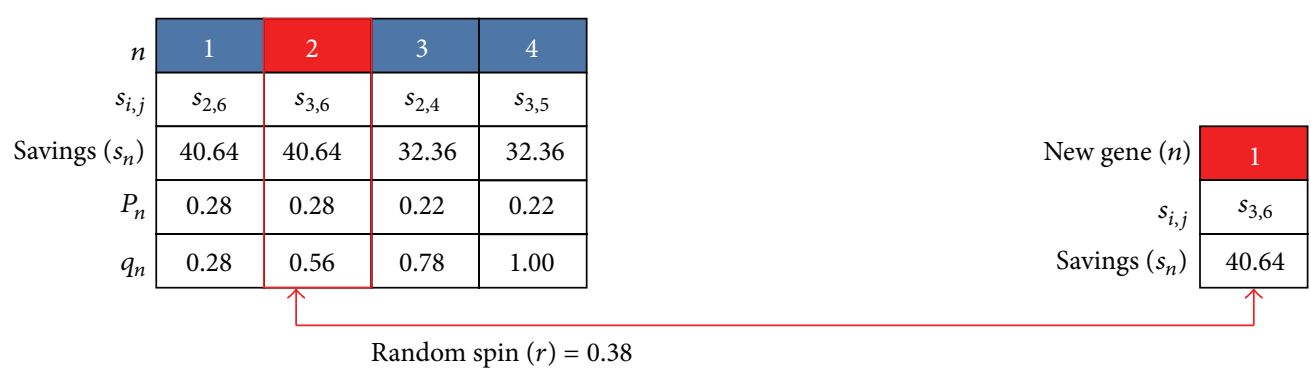

(b) $T=4$

\begin{tabular}{|c|c|c|c|c|c|c|c|c|c|}
\hline Gene $(n)$ & 1 & 2 & 3 & 4 & 5 & 6 & 7 & 8 & 9 \\
\hline$s_{i, j}$ & $s_{2,6}$ & $s_{2,4}$ & $s_{3,5}$ & $s_{2,3}$ & $s_{4,6}$ & $s_{5,6}$ & $s_{2,5}$ & $s_{3,4}$ & $s_{4,5}$ \\
\hline Savings $\left(s_{n}\right)$ & 40.64 & 32.36 & 32.36 & 30.58 & 28.28 & 28.28 & 20.00 & 20.00 & 11.72 \\
\hline
\end{tabular}

(c)

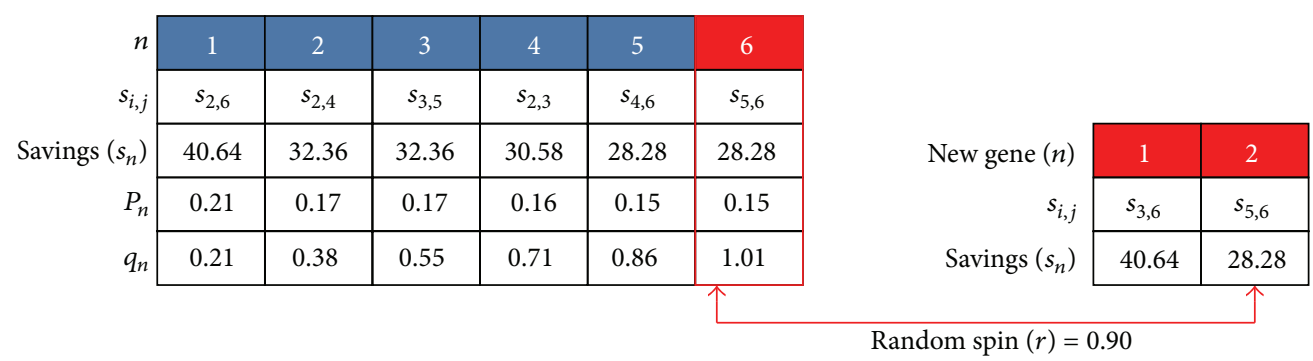

(d) $T=6$

\begin{tabular}{|c|c|c|c|c|c|c|c|c|c|c|}
\hline New gene $(n)$ & 1 & 2 & 3 & 4 & 5 & 6 & 7 & 8 & 9 & 10 \\
\hline$s_{i, j}$ & $s_{3,6}$ & $s_{5,6}$ & $s_{2,6}$ & $s_{2,4}$ & $s_{3,5}$ & $s_{2,3}$ & $s_{4,6}$ & $s_{2,5}$ & $s_{3,4}$ & $s_{4,5}$ \\
\hline Savings $\left(s_{n}\right)$ & 40.64 & 28.28 & 40.64 & 32.36 & 32.36 & 30.58 & 28.28 & 20.00 & 20.00 & 11.72 \\
\hline
\end{tabular}

(e)

FIGURE 5: The example of the two-phase selection procedure.

with savings value $\left(s_{n}\right)$ for each gene $(n)$ are calculated using the following equations:

$$
\begin{gathered}
p_{n}=\frac{s_{n}}{\sum_{i \in T} s_{i}} \quad \text { for } n \in T, \\
q_{n}=\sum_{i \in n} p_{i} \quad \text { for } n \in T .
\end{gathered}
$$

After that, we spin the wheel with a random number $(r=$ 0.38 ) from the range between 0 and 1 . The one savings value $\left(s_{2}\right)$ will be selected to be a gene of a new chromosome by considering $r$ and $q_{n}$. If $r \leq q_{1}$, then select the first savings value $s_{1}$; otherwise, select the $n$th savings value $s_{n}(2 \leq n \leq T)$ such that $q_{n-1}<r \leq q_{n}$. The selected gene is removed from the chromosome which leaves nine savings values as shown in Figure 5(c). Figure 5(d) shows the same selection process in the next iteration with parameters $T=6$ and $r=0.90$. Therefore, this procedure will be executed until the last gene of the chromosome is selected to be a gene of the new chromosome which is shown in Figure 5(e).

When the new chromosome which represents the new savings list is generated, it is calculated by the route merging procedure and the open-route construction procedure to produce a new OVRP solution. After we compare two solutions, the new chromosome will replace the previous chromosome only if the new solution is better than the 
previous one. This acceptance criterion is referred to a basic variable neighborhood search which is to accept only improvements [39]. Our approach is continued until the stopping criterion, which is the number of global iterations for two-phase selection, is satisfied.

2.4. The Route Postimprovement Procedure. In order to find any further improvements for the best solution found when the stopping criterion of the two-phase selection procedure is satisfied, we have developed the route postimprovement procedure to generate different routes in our best solution. In order to explore the whole neighborhood of our best solution, we focus on the order of customers in single route called intraroute and multiple routes called interroute. The neighborhood structures that we used are several well-known move operators found in the works of Subramanian et al. [35] and Groër et al. [36] including shift moves (1-0, 2-0, 3-0), swap moves (1-1, 2-1, 2-2), and $\lambda$-opt moves $(\lambda \in\{2,3\})$. The shift moves remove customers and insert them in another place. The swap moves select customers and exchange them. The $\lambda$-opt moves remove edges between customers and replace them with new edges. Our scheme is adapted from the local search strategy found in Çatay [40] by first applying a local neighborhood search to improve our best solution in each route. Then, a larger neighborhood search is applied across each pair between routes, respectively, by using all eight move operators with equal probability. This procedure is repeated until the stopping criterion, which is the number of consecutive iterations without any improvements in the best found solution, is satisfied.

\section{Computational Results}

The proposed CW was coded in Visual Basic 6.0 on an Intel Core i7 CPU 860 clocked at $2.80 \mathrm{GHz}$ with $1.99 \mathrm{~GB}$ of RAM under Windows XP platform. The numerical experiment used five well-known data sets of Euclidean benchmarks (composed of 62 instances) of the OVRP consisting of Augerat et al. [41] in data sets A, B, and P, Christofides and Eilon [42] in data set E, and Fisher [43] in data set F. The input data is available online at http://www.branchandcut.org/ (last access 1/2010). The best known solutions which are available online at http://www.hha.dk/ lys/ (last access 7/2011) are obtained by a branch-and-cut algorithm from the work of Letchford et al. [44]. In our approach, some parameters have to be preset before the execution as shown in Table 1 . Table 2 describes the development of the proposed CW in detail.

The benchmark problem sizes that we highlighted in this paper are classified as small-scale (less than 50 customers) and medium-scale (between 51 to 100 customers) with different features, for example, uniformly and not uniformly dispersed customers, clustered and not clustered, with a centered or not centered depot. All problems also include capacity constraints and minimum number of vehicles used restrictions. The first benchmark in data sets $\mathrm{A}, \mathrm{B}$, and $\mathrm{P}$ was proposed by Augerat et al. [41]. For the instances in data set A, both customer locations and demands are randomly generated. The customer locations in data set B are clustered
TABLE 1: The parameters used in the proposed CW.

\begin{tabular}{ll}
\hline Parameter & Values \\
\hline $\begin{array}{l}\text { The Clarke-Wright formula modification } \\
\text { procedure }\end{array}$ & \\
Route shape $(\lambda)$ & $\begin{array}{l}0.1-2.0 \\
\text { (increment of 0.1) }\end{array}$ \\
\hline $\begin{array}{l}\text { The two-phase selection procedure } \\
\text { Number of tournament sizes }\end{array}$ & $\begin{array}{l}3-20 \\
\text { (random number) }\end{array}$ \\
$\begin{array}{l}\text { Number of global iterations for two-phase } \\
\text { selection }\end{array}$ & 5,000
\end{tabular}

The route postimprovement procedure

Number of consecutive iterations without any

improvements in the best found solution

Probability to select each move operator

0.125

TABLE 2: The different features of the proposed CW.

\begin{tabular}{ll}
\hline Abbreviation & Details \\
\hline $\mathrm{CW}-1$ & $\begin{array}{l}\text { Improve CW solution with Clarke-Wright } \\
\text { formula modification procedure }\end{array}$ \\
$\mathrm{CW}-2$ & $\begin{array}{l}\text { Improve CW-1 solution with two-phase selection } \\
\text { procedure } \\
\text { Improve CW-2 solution with route } \\
\text { pW-3 }\end{array}$ \\
\hline
\end{tabular}

instances. The modified version of other instances is data set P. In these data sets, the problem ranges in size from 16 to 69 customers including the depot. The second benchmark in data set E was proposed by Christofides and Eilon [42]. In this data set, the customers are randomly distributed in the plane and the depot is either in the center or near to it. The problem ranges in size from 22 to 101 customers including the depot. The third benchmark in data set $\mathrm{F}$ is the real-life problem given by Fisher [43]. Instances F-n45-k4 and F-n135-k7 represent a day of grocery deliveries from the Peterboro and Bramalea, Ontario terminals, respectively, of National Grocers Limited. Instance F-n72-k4 represents the delivery of tires, batteries, and accessories to gasoline service stations. The depot is not centered in both instances. The problem ranges in size from 45 to 135 customers including the depot. We discuss each benchmark problem in which the percentage improvement between CW solution (cws) and obtained solution (obs) is calculated as follows:

$$
\text { Percentage improvement }=\left(\frac{\mathrm{cws}-\mathrm{obs}}{\mathrm{cws}}\right) \times 100 \text {. }
$$

Moreover, the percentage deviation between obtained solution (obs) and the best known solution (bks) is also calculated as follows:

$$
\text { Percentage deviation }=\left(\frac{\mathrm{obs}-\mathrm{bks}}{\mathrm{bks}}\right) \times 100 .
$$

The computational results for OVRP benchmark instances of Augerat et al. [41], Christofides and Eilon [42], and Fisher [43] are reported in Tables 3-5. We do not only consider the 
TABLE 3: Computational results between the proposed CW and CW for data sets A, B, P, E, C, and F.

\begin{tabular}{|c|c|c|c|c|c|c|c|c|c|c|}
\hline \multirow{2}{*}{ Number } & \multirow{2}{*}{ Instance } & \multicolumn{5}{|c|}{ Solution } & \multicolumn{3}{|c|}{ Percentage improvement } & \multirow{2}{*}{ CPU time (seconds) } \\
\hline & & CW & CW-1 & $\lambda$ & CW-2 & CW-3 & CW-1 & CW-2 & CW-3 & \\
\hline 1 & A-n32-k5 & 612.99 & 535.92 & 1.7 & 493.18 & 487.31 & 12.571 & 19.544 & 20.503 & 4.819 \\
\hline 2 & A-n33-k5 & 496.61 & 458.52 & 1.5 & 425.54 & 424.54 & 7.669 & 14.311 & 14.512 & 5.493 \\
\hline 3 & A-n33-k6 & 566.16 & 546.18 & 1.7 & 464.10 & 462.43 & 3.528 & 18.026 & 18.321 & 5.289 \\
\hline 4 & A-n34-k5 & 547.27 & 520.09 & 1.1 & 519.51 & 508.17 & 4.966 & 5.071 & 7.143 & 5.675 \\
\hline 5 & A-n36-k5 & 689.48 & 571.70 & 1.8 & 535.23 & 519.46 & 17.083 & 22.373 & 24.660 & 6.134 \\
\hline 6 & A-n37-k5 & 579.74 & 541.64 & 1.9 & 511.12 & 486.24 & 6.572 & 11.836 & 16.128 & 6.689 \\
\hline 7 & A-n37-k6 & 712.86 & 612.76 & 1.9 & 616.26 & 581.07 & 14.041 & 13.551 & 18.487 & 6.825 \\
\hline 8 & A-n38-k5 & 552.83 & 520.78 & 1.6 & 531.49 & 498.00 & 5.796 & 3.859 & 9.918 & 7.092 \\
\hline 9 & A-n39-k5 & 704.55 & 590.71 & 1.2 & 554.80 & 549.68 & 16.158 & 21.254 & 21.980 & 7.568 \\
\hline 10 & A-n39-k6 & 720.55 & 558.86 & 1.9 & 547.57 & 533.07 & 22.439 & 24.007 & 26.019 & 7.494 \\
\hline 11 & A-n44-k6 & 757.90 & 724.33 & 1.1 & 641.77 & 617.39 & 4.430 & 15.323 & 18.540 & 10.323 \\
\hline 12 & A-n45-k6 & 734.97 & 643.56 & 2.0 & 716.52 & 648.67 & 12.437 & 2.511 & 11.742 & 10.414 \\
\hline 13 & A-n45-k7 & 829.44 & 759.40 & 1.6 & 699.86 & 685.16 & 8.444 & 15.622 & 17.395 & 10.754 \\
\hline 14 & A-n46-k7 & 738.00 & 645.24 & 1.1 & 593.57 & 583.54 & 12.569 & 19.570 & 20.930 & 11.259 \\
\hline 15 & A-n48-k7 & 789.39 & 756.58 & 1.1 & 726.54 & 669.83 & 4.156 & 7.962 & 15.146 & 12.534 \\
\hline 16 & A-n53-k7 & 816.38 & 702.85 & 1.5 & 665.39 & 655.18 & 13.907 & 18.495 & 19.746 & 15.624 \\
\hline 17 & A-n54-k7 & 951.51 & 808.42 & 1.5 & 723.60 & 709.27 & 15.038 & 23.952 & 25.458 & 16.224 \\
\hline 18 & A-n55-k9 & 802.06 & 736.91 & 1.9 & 696.52 & 669.06 & 8.123 & 13.158 & 16.582 & 17.324 \\
\hline 19 & A-n62-k8 & 979.51 & 851.15 & 1.3 & 815.21 & 783.18 & 13.105 & 16.774 & 20.044 & 22.959 \\
\hline 20 & A-n65-k9 & 844.35 & 800.41 & 1.2 & 783.42 & 728.59 & 5.205 & 7.217 & 13.710 & 24.524 \\
\hline \multirow[t]{2}{*}{21} & A-n69-k9 & 942.87 & 798.64 & 2.0 & 773.17 & 757.76 & 15.296 & 17.998 & 19.632 & 28.600 \\
\hline & \multicolumn{6}{|c|}{ Average percentage improvement of data set A } & 10.644 & 14.877 & 17.933 & 11.601 \\
\hline 1 & B-n31-k5 & 383.68 & 367.00 & 0.9 & 364.80 & 362.73 & 4.347 & 4.923 & 5.463 & 4.365 \\
\hline 2 & B-n34-k5 & 541.34 & 506.26 & 0.9 & 459.59 & 458.76 & 6.480 & 15.103 & 15.254 & 5.556 \\
\hline 3 & B-n35-k5 & 599.16 & 595.15 & 1.4 & 567.34 & 557.33 & 0.669 & 5.311 & 6.982 & 5.981 \\
\hline 4 & B-n38-k6 & 500.64 & 483.14 & 1.4 & 450.72 & 445.63 & 3.495 & 9.972 & 10.989 & 7.109 \\
\hline 5 & B-n39-k5 & 382.11 & 354.03 & 1.4 & 334.70 & 322.54 & 7.350 & 12.409 & 15.590 & 7.602 \\
\hline 6 & B-n41-k6 & 539.15 & 507.25 & 1.8 & 493.34 & 483.07 & 5.917 & 8.496 & 10.402 & 8.549 \\
\hline 7 & B-n43-k6 & 483.21 & 481.57 & 1.6 & 432.30 & 428.17 & 0.340 & 10.536 & 11.391 & 9.422 \\
\hline 8 & B-n44-k7 & 575.52 & 560.54 & 1.9 & 512.64 & 501.31 & 2.603 & 10.927 & 12.895 & 10.244 \\
\hline 9 & B-n45-k5 & 601.71 & 512.73 & 1.9 & 509.56 & 488.07 & 14.788 & 15.315 & 18.887 & 10.357 \\
\hline 10 & B-n45-k6 & 459.90 & 430.83 & 1.1 & 431.54 & 403.81 & 6.323 & 6.168 & 12.197 & 10.573 \\
\hline 11 & B-n50-k7 & 537.23 & 491.93 & 1.9 & 446.07 & 437.15 & 8.433 & 16.969 & 18.629 & 13.265 \\
\hline 12 & B-n51-k7 & 703.81 & 683.31 & 2.0 & 656.01 & 625.14 & 2.913 & 6.792 & 11.178 & 13.509 \\
\hline 13 & B-n52-k7 & 482.90 & 465.17 & 1.8 & 450.07 & 441.19 & 3.672 & 6.798 & 8.637 & 14.586 \\
\hline 14 & B-n56-k7 & 497.15 & 474.13 & 1.5 & 463.06 & 420.48 & 4.629 & 6.856 & 15.420 & 17.409 \\
\hline 15 & B-n63-k10 & 950.48 & 950.48 & 1.0 & 857.90 & 837.07 & 0.000 & 9.740 & 11.931 & 23.724 \\
\hline 16 & B-n64-k9 & 572.41 & 541.70 & 1.2 & 581.72 & 520.47 & 5.364 & -1.627 & 9.074 & 23.764 \\
\hline \multirow[t]{2}{*}{17} & B-n68-k9 & 830.48 & 777.68 & 1.1 & 758.69 & 701.72 & 6.357 & 8.644 & 15.504 & 27.874 \\
\hline & \multicolumn{6}{|c|}{ Average percentage improvement of data set B } & 4.922 & 9.020 & 12.378 & 12.582 \\
\hline 1 & P-n16-k8 & 235.89 & 235.89 & 0.3 & 235.06 & 235.06 & 0.000 & 0.352 & 0.352 & 1.173 \\
\hline 2 & P-n19-k2 & 198.25 & 172.93 & 1.9 & 168.57 & 168.57 & 12.771 & 14.972 & 14.972 & 1.565 \\
\hline 3 & P-n20-k2 & 210.01 & 184.50 & 1.9 & 170.28 & 170.28 & 12.147 & 18.918 & 18.918 & 1.712 \\
\hline 4 & $\mathrm{P}-\mathrm{n} 21-\mathrm{k} 2$ & 209.92 & 180.27 & 1.8 & 168.15 & 163.88 & 14.124 & 19.897 & 21.933 & 1.729 \\
\hline
\end{tabular}


TABle 3: Continued.

\begin{tabular}{|c|c|c|c|c|c|c|c|c|c|c|}
\hline \multirow{2}{*}{ Number } & \multirow{2}{*}{ Instance } & \multicolumn{5}{|c|}{ Solution } & \multicolumn{3}{|c|}{ Percentage improvement } & \multirow{2}{*}{ CPU time (seconds) } \\
\hline & & CW & CW-1 & $\lambda$ & $\mathrm{CW}-2$ & CW-3 & CW-1 & CW-2 & CW-3 & \\
\hline 5 & P-n22-k2 & 206.00 & 183.58 & 1.8 & 171.46 & 167.19 & 10.883 & 16.765 & 18.840 & 1.888 \\
\hline 6 & P-n22-k8 & 370.26 & 345.53 & 1.6 & 352.14 & 345.87 & 6.679 & 4.892 & 6.588 & 2.330 \\
\hline 7 & P-n23-k8 & 309.74 & 307.28 & 1.7 & 304.83 & 302.87 & 0.794 & 1.586 & 2.219 & 2.608 \\
\hline 8 & P-n40-k5 & 420.65 & 395.73 & 1.5 & 370.64 & 349.55 & 5.924 & 11.889 & 16.902 & 7.749 \\
\hline 9 & P-n45-k5 & 459.29 & 442.23 & 1.5 & 396.64 & 391.81 & 3.714 & 13.641 & 14.692 & 10.159 \\
\hline 10 & P-n50-k7 & 468.42 & 447.69 & 1.5 & 440.56 & 397.38 & 4.426 & 5.948 & 15.167 & 13.237 \\
\hline 11 & P-n55-k7 & 513.45 & 464.90 & 1.4 & 452.69 & 411.58 & 9.455 & 11.833 & 19.840 & 16.627 \\
\hline 12 & P-n55-k8 & 505.72 & 476.43 & 1.8 & 442.21 & 412.55 & 5.793 & 12.558 & 18.423 & 16.349 \\
\hline 13 & P-n55-k10 & 555.25 & 502.56 & 1.6 & 488.65 & 444.31 & 9.490 & 11.995 & 19.981 & 17.387 \\
\hline 14 & P-n60-k10 & 584.40 & 539.43 & 1.8 & 503.38 & 482.09 & 7.694 & 13.864 & 17.507 & 20.499 \\
\hline \multirow[t]{2}{*}{15} & P-n65-k10 & 649.31 & 592.35 & 1.6 & 531.57 & 522.50 & 8.772 & 18.133 & 19.529 & 29.626 \\
\hline & \multicolumn{6}{|c|}{ Average percentage improvement of data set $\mathrm{P}$} & 7.511 & 11.816 & 15.058 & 9.642 \\
\hline 1 & E-n22-k4 & 286.91 & 260.61 & 1.3 & 252.61 & 252.61 & 9.169 & 11.955 & 11.955 & 3.753 \\
\hline 2 & E-n23-k3 & 497.18 & 456.86 & 1.2 & 444.29 & 442.98 & 8.111 & 10.638 & 10.901 & 4.156 \\
\hline 3 & E-n33-k4 & 633.04 & 576.29 & 1.4 & 518.04 & 511.26 & 8.965 & 18.165 & 19.236 & 12.223 \\
\hline 4 & E-n51-k5 & 493.02 & 477.78 & 2.0 & 452.67 & 416.06 & 3.091 & 8.185 & 15.610 & 36.154 \\
\hline 5 & E-n76-k10 & 697.03 & 641.48 & 1.9 & 587.35 & 567.14 & 7.971 & 15.736 & 18.635 & 68.917 \\
\hline \multirow[t]{2}{*}{6} & E-n101-k8 & 807.33 & 724.48 & 2.0 & 694.88 & 642.36 & 10.262 & 13.929 & 20.433 & 82.387 \\
\hline & \multicolumn{6}{|c|}{ Average percentage improvement of data set $\mathrm{E}$} & 7.928 & 13.101 & 16.128 & 34.598 \\
\hline 1 & F-n $45-\mathrm{k} 4$ & 615.12 & 535.85 & 2.0 & 478.40 & 463.90 & 12.886 & 22.227 & 24.584 & 10.365 \\
\hline 2 & F-n72-k4 & 208.29 & 191.18 & 2.0 & 187.67 & 177.00 & 8.212 & 9.899 & 15.021 & 42.554 \\
\hline \multirow[t]{2}{*}{3} & F-n135-k7 & 1033.24 & 856.33 & 1.9 & 816.30 & 775.80 & 17.122 & 20.996 & 24.916 & 112.258 \\
\hline & \multicolumn{6}{|c|}{ Average percentage improvement of data set $\mathrm{F}$} & 12.740 & 17.707 & 21.507 & 55.059 \\
\hline
\end{tabular}

Italic number indicates the infeasible solution (the number of vehicles used is inadequate).

TABLE 4: Computational results between the proposed CW and MA for data sets A, P, and F.

\begin{tabular}{|c|c|c|c|c|c|c|}
\hline \multirow{2}{*}{ Number } & \multirow{2}{*}{ Instance } & \multicolumn{3}{|c|}{ Solution } & \multicolumn{2}{|c|}{ Percentage deviation } \\
\hline & & Best Known & MA & Our CW & MA & Our CW \\
\hline 1 & A-n32-k5 & 487.31 & 487.31 & 487.31 & 0.000 & 0.000 \\
\hline 2 & A-n33-k5 & 424.54 & 424.54 & 424.54 & 0.000 & 0.000 \\
\hline 3 & A-n33-k6 & 462.43 & 462.43 & 462.43 & 0.000 & 0.000 \\
\hline 4 & A-n34-k5 & 508.17 & 508.52 & 508.17 & 0.068 & 0.000 \\
\hline 5 & A-n36-k5 & 519.46 & 519.46 & 519.46 & 0.000 & 0.000 \\
\hline 6 & A-n37-k5 & 486.24 & 486.24 & 486.24 & 0.000 & 0.000 \\
\hline 7 & P-n19-k2 & 168.57 & 168.57 & 168.57 & 0.000 & 0.000 \\
\hline 8 & P-n20-k2 & 170.28 & 170.28 & 170.28 & 0.000 & 0.000 \\
\hline 9 & P-n21-k2 & 163.88 & 163.88 & 163.88 & 0.000 & 0.000 \\
\hline 10 & P-n22-k2 & 167.19 & 167.19 & 167.19 & 0.000 & 0.000 \\
\hline 11 & P-n40-k5 & 349.55 & 349.55 & 349.55 & 0.000 & 0.000 \\
\hline 12 & P-n45-k5 & 391.81 & 391.81 & 391.81 & 0.000 & 0.000 \\
\hline 13 & P-n50-k7 & 397.38 & 407.73 & 397.38 & 2.605 & 0.000 \\
\hline 14 & F-n $45-\mathrm{k} 4$ & 463.90 & 463.90 & 463.90 & 0.000 & 0.000 \\
\hline \multirow[t]{2}{*}{15} & F-n72-k4 & 177.00 & 177.45 & 177.00 & 0.257 & 0.000 \\
\hline & \multicolumn{4}{|c|}{ Average percentage deviation of data sets $\mathrm{A}, \mathrm{P}$, and $\mathrm{F}$} & 0.195 & 0.000 \\
\hline
\end{tabular}


TABLE 5: Computational results between the proposed CW and the other algorithms for data sets E and F.

\begin{tabular}{|c|c|c|c|c|c|c|c|}
\hline \multirow{2}{*}{ Number } & \multirow{2}{*}{ Instance } & \multicolumn{6}{|c|}{ Solution } \\
\hline & & Best Known & B & $\mathrm{PR}$ & LGW & $\mathrm{FOH}$ & Our CW \\
\hline 1 & E-n51-k5 & 416.06 & 416.1 & 416.06 & 416.06 & 416.06 & 416.06 \\
\hline 2 & E-n76-k10 & 567.14 & 574.5 & 567.14 & 567.14 & 567.14 & 567.14 \\
\hline 3 & E-n101-k8 & 639.74 & 641.6 & 641.76 & 639.74 & 639.74 & 642.36 \\
\hline 4 & F-n72-k4 & 177.00 & 177.4 & 177.00 & 177.00 & 178.09 & 177.00 \\
\hline 5 & F-n135-k7 & 769.66 & 781.2 & 770.17 & 769.66 & 769.66 & 775.80 \\
\hline \multirow{2}{*}{ Number } & \multirow{2}{*}{ Instance } & & \multicolumn{5}{|c|}{ CPU time (seconds) } \\
\hline & & & B & PR & LGW & $\mathrm{FOH}$ & Our CW \\
\hline 1 & E-n51-k5 & & 88.8 & 230 & 6.2 & 17.6 & 36.154 \\
\hline 2 & E-n76-k10 & & 167.5 & 530 & 31.3 & 29.0 & 68.917 \\
\hline 3 & E-n101-k8 & & 325.3 & 1280 & 39.5 & 239.6 & 82.387 \\
\hline 4 & F-n72-k4 & & 398.1 & 1040 & 19.5 & 140.2 & 42.554 \\
\hline 5 & F-n135-k7 & & 1000.2 & 3590 & 158.2 & 1237.5 & 112.258 \\
\hline
\end{tabular}

Bold number indicates the best known solution was obtained.

improvements of our solutions over CW solutions as shown in Table 3, but also show the performance of our solutions by comparing the proposed CW with the algorithms for OVRP as shown in Tables 4 and 5 by using the following abbreviations: MA for Mirhassani and Abolghasemi [21], B for Brandão [14], PR for Pisinger and Ropke [45], LGW for Li et al. [46] and FOH for Fleszar et al. [20]. Results from Tables 3-5 indicate that the proposed CW can find high quality solutions within reasonable time, especially for small and medium scale problems. Out of 62 problems, we find the optimal solutions for 60 problems with up to 134 customers. For two problems with 100-134 customers, the percentage deviations between our solutions and the optimal solutions are very low (E-n101-k8 and F-n135-k7). Nevertheless, in those optimal and near optimal solutions, there are four problems (A-n34-k5, P-n50-k7, F-n72-k4, and E-n76-k10) for which our results in Tables 4 and 5 are better than the others. Moreover, our results in Table 3 show that the proposed CW always performed better than CW. These indicate that the proposed CW is effective and efficient in producing high quality solutions for well-known benchmark problems. The important details of our improvement are discussed below.

The average percentage improvements between CW solutions and our solutions for benchmark of data sets A, B, P, E, and $\mathrm{F}$ are presented in Table 3. We have found that CW solutions were improved by the average of $15.712 \%$. This finding shows that data set $\mathrm{F}$ has the highest average improvement and data set $\mathrm{B}$ has the lowest average deviation. The greatest improvements of CW solutions are, respectively, presented in top three instances including A-n39-k6 (26.019\%), A-n54-k7 (25.458\%), and F-n135-k7 (24.916\%). We can conclude that the problems which have the features like clustered customers can be solved by CW better than the problems which have the features like dispersed customers. In addition, the results show that the proposed CW can solve both above-mentioned problems to obtain optimal or near optimal solutions.

The performance of route postimprovement procedure can be described as the reduction of the percentage deviation between CW-2 and CW-3 solutions. The average reduction of the percentage deviation between $\mathrm{CW}-2$ solutions and $\mathrm{CW}$ 3 solutions for benchmark of data sets A, B, P, E, and F are $3.652,3.857,3.938,3.699$, and 4.806. We have found that CW-3 solutions were reduced by the average of $3.838 \%$. The greatest reductions of CW-3 solutions are, respectively, presented in top three instances including B-n64-k9 (11.769\%), P-n50-k7 (10.866\%), and A-n45-k6 (10.460\%). According to Table 3, some CW-3 solutions can be reduced to obtain the optimal solutions.

Another finding from our work is the infeasible solutions produced by $\mathrm{CW}$ in which the number of vehicles used is inadequate. This finding is referred to Vigo [47] that CW does not allow for the control of the number of routes of the final solution. The solution found for a given instance can, in fact, require more than $k$ routes to serve all the customers, hence being infeasible.

\section{Conclusions}

In this paper, we have presented a new heuristic approach based on Clarke-Wright algorithm to solve the open vehicle routing problem (OVRP). We have modified the ClarkeWright algorithm with three procedures composed of ClarkeWright formula modification, open-route construction, and two-phase selection and have combined them with a route postimprovement procedure in which the neighborhood structures composed of shift, swap, and $\lambda$-opt move operators are used to improve our best solution. We also have done experiments using six well-known data sets of OVRP (composed of 62 instances) obtained from the literatures. The numerical results show that our approach is competitive and our solutions outperform Clarke and Wright [22] in all directions. Moreover, it also generates the best known solutions in $97 \%$ of all instances (60 out of 62 ).

During the development of our approach, we have mentioned the ideas related to the $\mathrm{CW}$ that deserve more attention in further studies. Consequently, it may be interesting to develop a more powerful postimprovement procedure. An additional study is to extend the proposed CW to deal with 
other variants of the studied problems such as simultaneous pickup and delivery (VRPSPD) or time windows (VRPTW).

\section{References}

[1] D. Sariklis and S. Powell, "A heuristic method for the open vehicle routing problem," The Journal of the Operational Research Society, vol. 51, no. 5, pp. 564-573, 2000.

[2] Z. Fu and M. Wright, "Train plan model for British rail freight services through the channel tunnel," The Journal of the Operational Research Society, vol. 45, no. 4, pp. 384-391, 1994.

[3] L. Y. O. Li and Z. Fu, "The school bus routing problem: a case study," The Journal of the Operational Research Society, vol. 53, no. 5, pp. 552-558, 2002.

[4] C. D. Tarantilis and C. T. Kiranoudis, "Distribution of fresh meat," Journal of Food Engineering, vol. 51, no. 1, pp. 85-91, 2002.

[5] R. Russell, W.-C. Chiang, and D. Zepeda, "Integrating multiproduct production and distribution in newspaper logistics," Computers \& Operations Research, vol. 35, no. 5, pp. 1576-1588, 2008.

[6] W.-C. Chiang, R. Russell, X. Xu, and D. Zepeda, "A simulation/metaheuristic approach to newspaper production and distribution supply chain problems," International Journal of Production Economics, vol. 121, no. 2, pp. 752-767, 2009.

[7] P. P. Repoussis, D. C. Paraskevopoulos, G. Zobolas, C. D. Tarantilis, and G. Ioannou, "A web-based decision support system for waste lube oils collection and recycling," European Journal of Operational Research, vol. 195, no. 3, pp. 676-700, 2009.

[8] S. Yu, C. Ding, and K. Zhu, "A hybrid GA-TS algorithm for open vehicle routing optimization of coal mines material," Expert Systems with Applications, vol. 38, no. 8, pp. 10568-10573, 2011.

[9] N. Christofides, "Worst-case analysis of a new heuristic for the travelling salesman problem," Tech. Rep. 388, Graduate School of Industrial Administration, Canegie Mellon University, 1976.

[10] M. Held and R. M. Karp, "The traveling salesman problem and minimum spanning trees," Operations Research, vol. 18, pp. $1138-1162,1970$.

[11] R. E. Miller and J. W. Thatcher, Complexity of Computer Computations, Plenum Press, New York, NY, USA, 1972.

[12] M. Syslo, N. Deo, and J. Kowaklik, Discrete Optimization Algorithms with Pascal Programs, Prentice Hall, Englewood Cliffs, NJ, USA, 1983.

[13] P. Toth and D. Vigo, The Vehicle Routing Problem, SIAM Monographs on Discrete Mathematics and Applications, SIAM Publishing, Philadelphia, Pa, USA, 2002.

[14] J. Brandão, "A tabu search algorithm for the open vehicle routing problem," European Journal of Operational Research, vol. 157, no. 3, pp. 552-564, 2004.

[15] M. Salari, P. Toth, and A. Tramontani, "An ILP improvement procedure for the Open Vehicle Routing Problem," Computers \& Operations Research, vol. 37, no. 12, pp. 2106-2120, 2010.

[16] G. B. Dantzig and J. H. Ramser, "The truck dispatching problem," Management Science, vol. 6, pp. 80-91, 1959.

[17] C. D. Tarantilis, D. Diakoulaki, and C. T. Kiranoudis, "Combination of geographical information system and efficient routing algorithms for real life distribution operations," European Journal of Operational Research, vol. 152, no. 2, pp. 437-453, 2004.

[18] Z. Fu, R. Eglese, and L. Li, "A new tabu search heuristic for the open vehicle routing problem," The Journal of the Operational Research Society, vol. 56, no. 3, pp. 267-274, 2005.
[19] X. Li and P. Tian, "An ant colony system for the open vehicle routing problem," in Ant Colony Optimization and Swarm Intelligence, M. Dorigo, L. M. Gambardella, M. Birattari, A. Martinoli, R. Poli, and T. Stützle, Eds., vol. 4150 of Lecture Notes in Computer Science, pp. 356-363, Springer, Berlin, Germany, 2006.

[20] K. Fleszar, I. H. Osman, and K. S. Hindi, "A variable neighbourhood search algorithm for the open vehicle routing problem," European Journal of Operational Research, vol. 195, no. 3, pp. 803-809, 2009.

[21] S. A. Mirhassani and N. Abolghasemi, "A particle swarm optimization algorithm for open vehicle routing problem," Expert Systems with Applications, vol. 38, no. 9, pp. 11547-11551, 2011.

[22] G. Clarke and J. W. Wright, "Scheduling of vehicles from a central depot to a number of delivery points," Operations Research, vol. 12, pp. 568-581, 1964.

[23] F. A. Tillman, "The multiple terminal delivery problem with probabilistic demands," Transportation Science, vol. 3, pp. 192204, 1969.

[24] L. Bodin, B. Golden, A. Assad, and M. Ball, "Routing and scheduling of vehicles and crews. The state of the art," Computers \& Operations Research, vol. 10, no. 2, pp. 63-211, 1983.

[25] I. Deif and L. D. Bodin, "Extension of the Clarke-Wright algorithm for solving the vehicle routing problem with backhauling," in Proceedings of the Babson Conference on Software Uses in Transportation and Logistics Management, A. E. Kidder, Ed., pp. 75-96, Babson Park, Mass, USA, 1984.

[26] T. J. Gaskell, "Bases for vehicle fleet scheduling," Operational Research Quarterly, vol. 18, pp. 281-295, 1967.

[27] P. Yellow, "A computational modification to the savings method of vehicle scheduling," Operational Research Quarterly, vol. 21, pp. 281-283, 1970.

[28] H. Paessens, "The savings algorithm for the vehicle routing problem," European Journal of Operational Research, vol. 34, no. 3, pp. 336-344, 1988.

[29] İ. K. Altinel and T. Öncan, "A new enhancement of the Clarke and Wright savings heuristic for the capacitated vehicle routing problem," The Journal of the Operational Research Society, vol. 56, no. 8, pp. 954-961, 2005.

[30] T. Doyuran and B. Çatay, "A robust enhancement to the ClarkeWright savings algorithm," The Journal of the Operational Research Society, vol. 62, no. 1, pp. 223-231, 2011.

[31] A. A. Juan, J. Faulin, R. Ruiz, B. Barrios, and S. Caballé, “The SR-GCWS hybrid algorithm for solving the capacitated vehicle routing problem," Applied Soft Computing Journal, vol. 10, no. 1, pp. 215-224, 2010.

[32] A. A. Juan, J. Faulin, J. Jorba, D. Riera, D. Masip, and B. Barrios, "On the use of Monte Carlo simulation, cache and splitting techniques to improve the Clarke and Wright savings heuristics," The Journal of the Operational Research Society, vol. 62, no. 6, pp. 1085-1097, 2011.

[33] T. Pichpibul and R. Kawtummachai, "New enhancement for Clarke-Wright savings algorithm to optimize the capacitated vehicle routing problem," European Journal of Scientific Research, vol. 78, pp. 119-134, 2012.

[34] T. Pichpibul and R. Kawtummachai, "An improved Clarke and Wright savings algorithm for the capacitated vehicle routing problem," ScienceAsia, vol. 38, pp. 307-318, 2012.

[35] A. Subramanian, L. M. A. Drummond, C. Bentes, L. S. Ochi, and R. Farias, "A parallel heuristic for the vehicle routing 
problem with simultaneous pickup and delivery," Computers \& Operations Research, vol. 37, no. 11, pp. 1899-1911, 2010.

[36] C. Groër, B. Golden, and E. Wasil, "A library of local search heuristics for the vehicle routing problem," Mathematical Programming Computation, vol. 2, no. 2, pp. 79-101, 2010.

[37] G. Laporte, M. Gendreau, J. Potvin, and F. Semet, "Classical and modern heuristics for the vehicle routing problem," International Transactions in Operational Research, vol. 7, no. 4-5, pp. 285-300, 2000.

[38] M. Gen and R. Cheng, Genetic Algorithms and Engineering Design, John Wiley \& Sons, New York, NY, USA, 1997.

[39] V. C. Hemmelmayr, K. F. Doerner, and R. F. Hartl, "A variable neighborhood search heuristic for periodic routing problems," European Journal of Operational Research, vol. 195, no. 3, pp. 791-802, 2009.

[40] B. Çatay, "A new saving-based ant algorithm for the vehicle routing problem with simultaneous pickup and delivery," Expert Systems with Applications, vol. 37, no. 10, pp. 6809-6817, 2010.

[41] P. Augerat, J. Belenguer, E. Benavent, A. Corbern, D. Naddef, and G. Rinaldi, "Computational results with a branch and cut code for the capacitated vehicle routing problem," Research Report 949-M, Universite Joseph Fourier, Grenoble, France, 1995.

[42] N. Christofides and S. Eilon, "An algorithm for the vehicle dispatching problem," Operational Research Quarterly, vol. 20, pp. 309-318, 1969.

[43] M. L. Fisher, "Optimal solution of vehicle routing problems using minimum K-trees," Operations Research, vol. 42, no. 4, pp. 626-642, 1994.

[44] A. N. Letchford, J. Lysgaard, and R. W. Eglese, "A branch-andcut algorithm for the capacitated open vehicle routing problem," The Journal of the Operational Research Society, vol. 58, no. 12, pp. 1642-1651, 2007.

[45] D. Pisinger and S. Ropke, "A general heuristic for vehicle routing problems," Computers \& Operations Research, vol. 34, no. 8, pp. 2403-2435, 2007.

[46] F. Li, B. Golden, and E. Wasil, "The open vehicle routing problem: algorithms, large-scale test problems, and computational results," Computers \& Operations Research, vol. 34, no. 10, pp. 2918-2930, 2007.

[47] D. Vigo, "Heuristic algorithm for the asymmetric capacitated vehicle routing problem," European Journal of Operational Research, vol. 89, no. 1, pp. 108-126, 1996. 

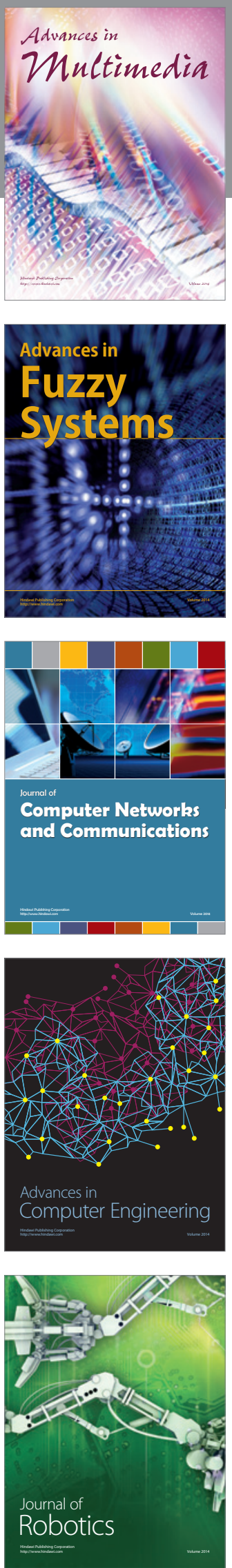

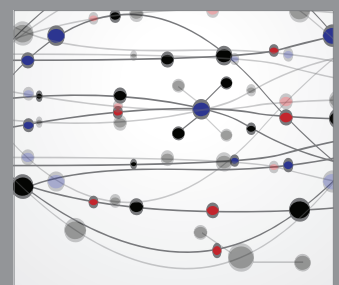

The Scientific World Journal
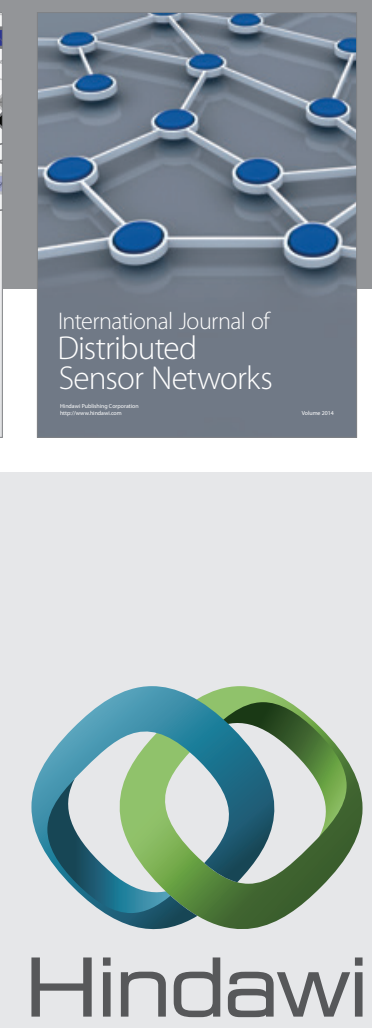

Submit your manuscripts at

http://www.hindawi.com
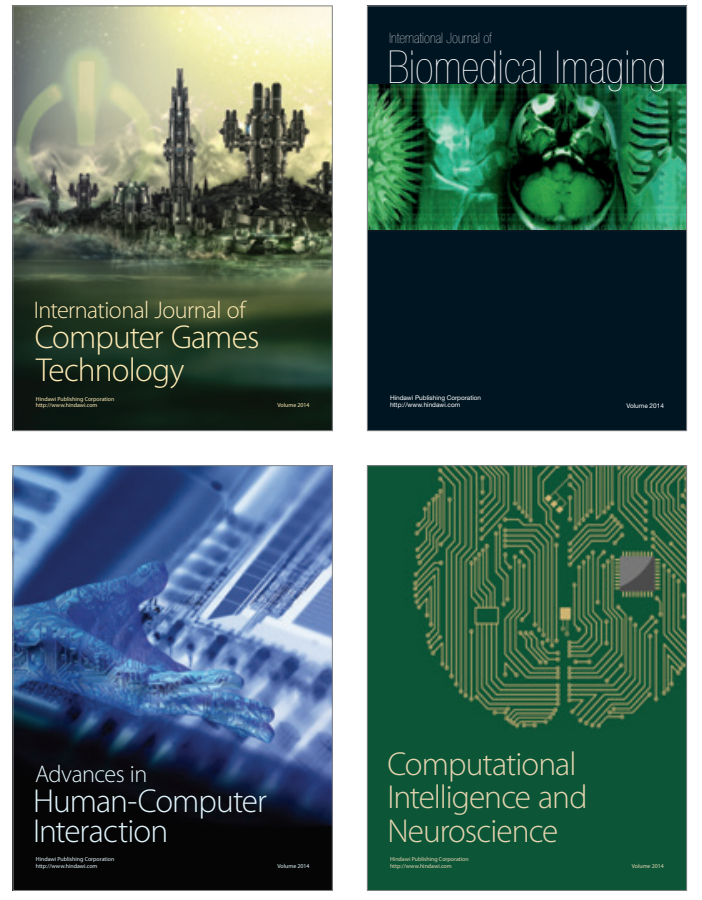
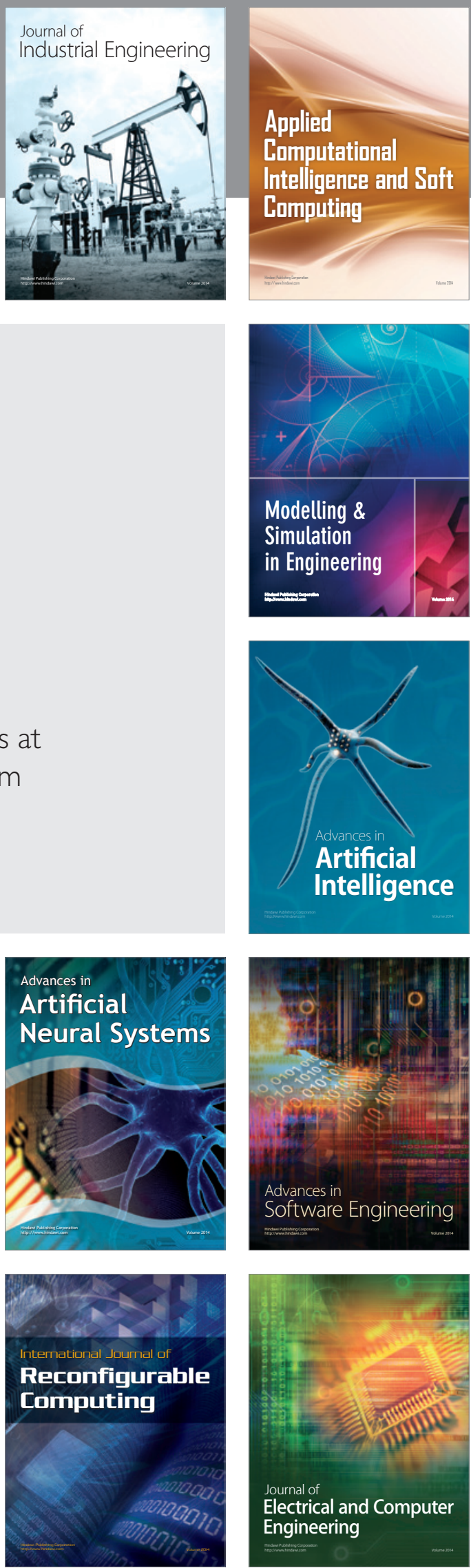